\title{
PROGRESS IN HEAVY ION DRIVEN INERTIAL FUSION ENERGY: FROM SCALED EXPERIMENTS TO THE INTEGRATED RESEARCH EXPERIMENT*
}

J. J. Barnard ${ }^{1}$, L. E. Ahle ${ }^{1}$, D. Baca ${ }^{2}$, R. O. Bangerter ${ }^{2}$, F. M. Bieniosek ${ }^{2}$, C. M. Celata ${ }^{2}$, E. Chacon-Golcher ${ }^{2}$, R. C. Davidson $^{3}$, A. Faltens ${ }^{2}$, A. Friedman ${ }^{1}$, R.M. Franks ${ }^{2}$, D. P. Grote ${ }^{1}$, I. Haber ${ }^{4}$, E. Henestroza ${ }^{2}$, M. J. L. de Hoon ${ }^{2}$, I. Kaganovich $^{3}$, V. P. Karpenko' ${ }^{1}$, R. A. Kishek ${ }^{5}$, J. W. Kwan ${ }^{2}$, E. P. Lee ${ }^{2}$, B. G. Logan ${ }^{1}$, S.M. Lund ${ }^{1}$, W. R. Meier ${ }^{1}$, A.

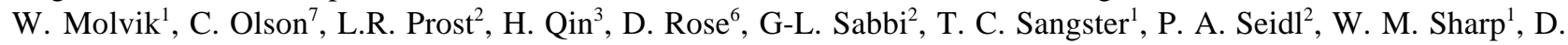
Shuman $^{2}$, J.L. Vay ${ }^{2}$, W.L. Waldron ${ }^{2}$, D. Welch ${ }^{6}$, S.S. $\mathrm{Yu}^{2}$

1. Lawrence Livermore National Laboratory, Livermore, CA; 2. Lawrence Berkeley National Laboratory, Berkeley, CA; 3. Princeton Plasma Physics Laboratory, Princeton, NJ; 4. Naval Research Laboratory, Washington, D.C.;

5. University of Maryland, College Park, MD 6.Mission Research Corporation, Albuquerque, NM 7.Sandia

National Laboratory, Albuquerque, NM

\section{Abstract}

The promise of inertial fusion energy driven by heavy ion beams requires the development of accelerators that produce ion currents $(\sim 100$ 's Amperes/beam) and ion energies $(\sim 1-10 \mathrm{GeV})$ that have not been achieved simultaneously in any existing accelerator. The high currents imply high generalized perveances, large tune depressions, and high space charge potentials of the beam center relative to the beam pipe. Many of the scientific issues associated with ion beams of high perveance and large tune depression have been addressed over the last two decades on scaled experiments at Lawrence Berkeley and Lawrence Livermore National Laboratories, the University of Maryland, and elsewhere. The additional requirement of high space charge potential (or equivalently high line charge density) gives rise to effects (particularly the role of electrons in beam transport) which must be understood before proceeding to a large scale accelerator. The first phase of a new series of experiments in the Heavy Ion Fusion Virtual National Laboratory (HIF VNL), the High Current Experiments (HCX), is now beginning at LBNL. The mission of the HCX is to transport beams with driver line charge density so as to investigate the physics of this regime, including constraints on the maximum radial filling factor of the beam through the pipe. This factor is important for determining both cost and reliability of a driver scale accelerator. The HCX will provide data for design of the next steps in the sequence of experiments leading to an inertial fusion energy power plant. The focus of the program after the HCX will be on integration of all of the manipulations required for a driver. In the near term following HCX, an Integrated Beam Experiment (IBX) of the same general scale as the HCX is envisioned.

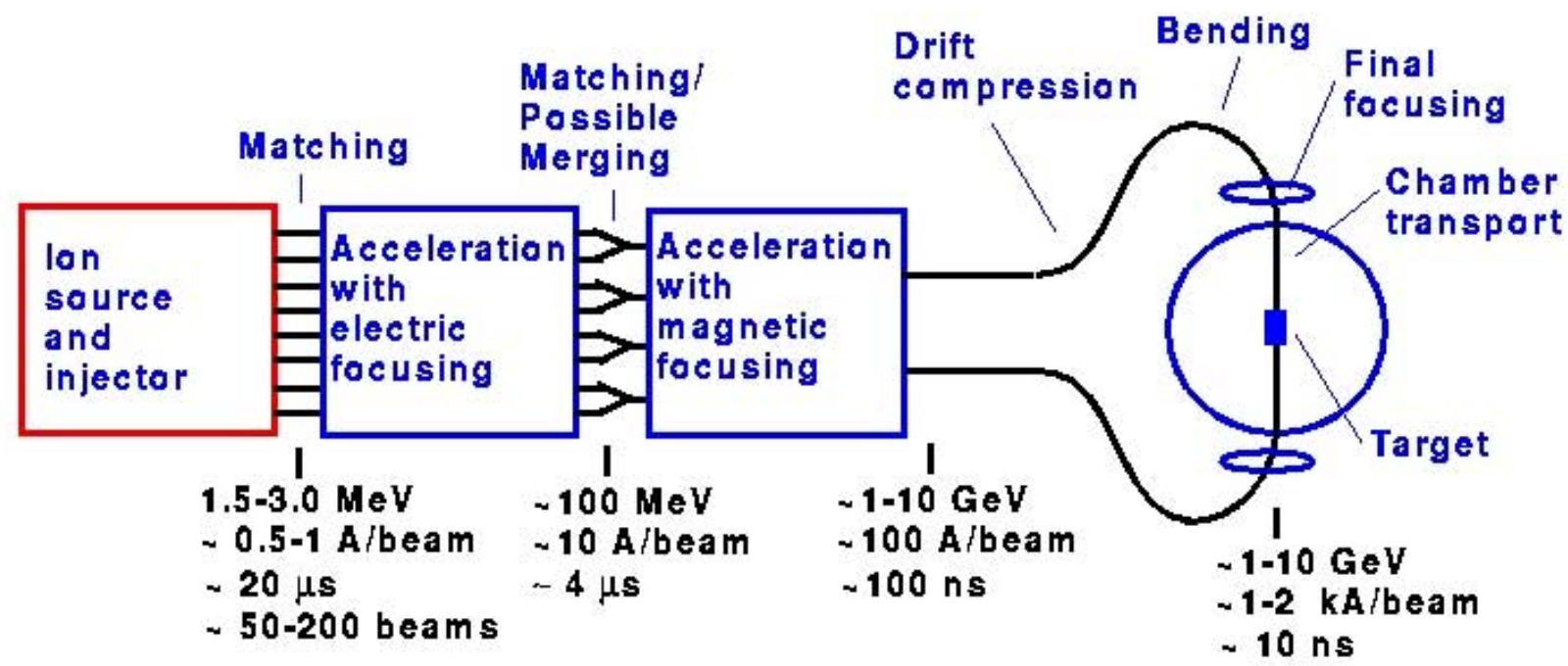

Figure 1. Schematic of the stages and beam manipulations required in one version of a Heavy Ion Fusion driver.

\footnotetext{
* This work was performed under the auspices of the U.S. Department of Energy under University of California contract W-7405-ENG-48 at LLNL, and University of California contract DE-AC03-76SF00098 at LBNL, and PPPL contract
} 
The step which bridges the gap between the IBX and an engineering test facility for fusion has been designated the Integrated Research Experiment (IRE). The IRE (like the IBX) will provide an integrated test of the beam physics necessary for a driver, but in addition will provide target and chamber data. This paper will review the experimental and theoretical progress in heavy ion accelerator driver research from the scaled experiments through the present experiments and will discuss plans for the IRE.

\section{INTRODUCTION}

Inertial fusion energy targets require the deposition of beam energy onto small spots, 2 to $5 \mathrm{~mm}$ in radius, at the ends of a hohlraum (indirect drive) or onto a spherical capsule (direct drive). The most detailed radiation/hydrodynamical simulations of heavy ion targets have been done for hohlraums in which the ions deposit their energy in converters. The converters radiate their energy in x-rays, which impinge upon capsules, ablating and compressing them[1]. The total pulse energy required is $\sim 3$ to $7 \mathrm{MJ}$, with a main pulse duration of $\sim 8-10 \mathrm{~ns}$. The ion range required by the target is $\sim .02$ to $0.20 \mathrm{~g} / \mathrm{cm}^{2}$, which implies an ion energy of between $\sim 1$ to $10 \mathrm{GeV}$ for ion masses between $\sim 80$ and 200. Final ion currents between 30 and $900 \mathrm{kA}$ are thus needed to meet the pulse energy requirement. The high currents are achieved by compressing the beam length by a factor of order 20 , so initial line charge densities between 15 and $900 \mu \mathrm{C} / \mathrm{m}$ are required. Quadrupole channels can transport a fraction of a $\mu \mathrm{C} / \mathrm{m}$ at typical injection energies. Comparing what is required at the target with the transportable current illustrates the need for multiple beams (10's to 100 's). Figure 1 illustrates the manipulations envisioned in a heavy ion fusion driver. These include injection of multiple beams, electric transport, a possible merge before magnetic transport, drift compression, beam bending, final focusing, and neutralized chamber transport .

The issues facing HIF can be broadly classified into two main groups: cost and focusability. The cost issue is continually being addressed both through studying component scaling imposed by the physics of beam transport and acceleration as well as through technology development projects required for the near term experiments (such as superconducting magnets, induction core materials, and insulators.) System studies are also used to determine high leverage machine architecture items affecting the overall cost of electricity.

The main scientific issue is focusability on the target. There are two main components which act to prevent focusability at the target: 1 . Space charge: Because currents are large, and because the chamber environment is envisioned to be filled with residual gas at the millitorr level, the mainline approach is to ionize the gas at the entrance to the target chamber. In addition, photoionization of the chamber gas by the beam heated target X-rays can be utilized. In both cases, the beam will draw electrons into its path to neutralize the space charge. Experiments and calculations are validating this concept.

2. Insufficient brightness: Over most of the beam path through the accelerator, economics dictates that space charge forces be much greater than thermal forces. At the target when the beam is focused down to a small spot thermal forces dominate (particularly when the beam is neutralized). It is thus important to maintain low emittance beams throughout, even when the emittance is not dynamically important. By making small, intense beams, target energy requirements can be reduced implying smaller, cheaper accelerators. So there is a big impact for getting the brightest beams possible.

\section{EXPERIMENTAL PROGRAM}

\subsection{Scaled Experiments[10]}

Two flagship experiments of the HIF program in the mideighties and early nineties began the process of demonstrating that the manipulations required for an induction linac driver can be carried out.

In the Single Beam Transport Experiment (SBTE) [3] (mid-1980's), the limits in undepressed and depressed phase advance were investigated. In contrast to some theoretical predictions of instabilities (using a KV distribution function), particle-in-cell simulations predicted and SBTE results then confirmed stable propagation for tune depressions (ratio of depressed to undepressed phase advance) as low as .1 to .2 (the limit achievable in the experiment). Undepressed phase advances greater than $\sim 85$ degrees were unstable for any significant tune depressions. Further, the experiment was at a length that was a significant fraction of the number of half-lattice periods in a driver $(\sim 10 \%)$.

The Multiple Beam Experiment (MBE-4) [4] (late 1980's and early 1990's) provided an initial demonstration of acceleration, current amplification, longitudinal confinement using "ear" fields, and transport of multiple (i.e. four) beams.

A bending experiment at LLNL [5], demonstrated bending and acceleration using induction cells, sensing (using capacitive probes, even while firing the induction cells) and steering the beam. The original plan was to make the bend the beginning of a recirculating induction accelerator, but limited funding forced the program to concentrate on the mainline linac approach.

More recently [6] it was demonstrated that the four beams from MBE4 could be combined into a single beam, with an emittance growth that was expected on the basis of both theory and simulation.

Also, recently [7] the SBTE apparatus was again used to ballistically focus a beam using a one-tenth scale version of a final focus design from the power plant study called 
HIBALL II [28]. Electric quads were used to prepare the beam for entrance into a magnetic final focus system. A spot size consistent with space charge and emittance was produced. In a later version of the experiment a heated wire filament was placed in the beam path supplying the beam with neutralizing electrons. Simulations using the LSP code agreed well with the experiment [8].

Other scaled experiments include an adiabatic plasma lens [9], a channel transport experiment [9] and others[10]. including the University of Maryland electron beam experiments [11], which are highly relevant to HIF.

Some of the HIF experiments (past, present, and future) are summarized in Table 1. It can be seen from the table that nearly every major manipulation required in an HIF driver, has been carried out, at some level, in the scaled experiments.

\subsection{Driver Scale Experiments}

Presently, the HIF program is developing experiments in which the line charge density of the beam is at or near that expected for the early phase of a driver accelerator. Line charge density is important, because it determines the space charge potential drop of the beam from center to the pipe radius, and hence the confining potential for both unwanted electrons (in the accelerator) and wanted electrons (in the chamber). Hence, the present program is examining the science of the propagation of "driver-scale" beams.

One of the main focuses of the program is the High Current experiment (HCX) [12]. The first phase of the experiment (next $\sim 2$ years) is to transport a driver scale beam $\left(\sim 0.6\right.$ ampere and $\sim 1.8 \mathrm{MeV}$ of $\left.\mathrm{K}^{+}\right)$. The potential drop from beam center to pipe radius will be $\sim 5 \mathrm{keV}$. This phase of the experiment will consist of 40 electrostatic quadrupoles followed by four magnetic to begin assessing magnetic transport. The second phase is currently planned to examine transport through 50-100 magnetic quads.
The beam filling factor $r_{\text {beam }} / r_{\text {pipe }}$ is important for obtaining a cost-optimized accelerator. Understanding the evolution of the emittance has strong implications for the target, and thus on overall cost. So the first phase of HCX will be to assess how close the beam can come to the pipe, by observing emittance growth, halo formation, and beam loss. The beam radius will be altered by changing the quadrupole voltages as well as the current. The question of optimum steering will be addressed, as well as the rate of electron production and entrainment. The role of desorbed atoms, born from beam halo particles hitting the walls or from ionized residual gas atoms accelerated by the beam space charge towards the wall, will be assessed. Pulse duration limits (within the bounds of the experiment) from head particle loss affecting tail propagation will be explored.

Phase 1 will use the existing ESQ injector and matching section. (The matching section reduces the radius of the beam out of the diode and injector, and transforms it from a circular to an elliptical beam). This will be followed by four 10 quad blocks. At the beginning of each block there will be a quad which slides out of the way, so that diagnostics such as slit-scanners or pepper-pots can be inserted into the beam path. Further, two of the quads in each 10 quad block can be displaced in $\mathrm{x}$ and $\mathrm{y}$, producing a dipole component, and allowing steering experiments that place the beam closer to the wall. The first four quads in each block allow independent control of their voltages so that the beam can be rematched if necessary or envelope oscillations intentionally induced to examine halo production. Finally, one quad in each block can be intentionally rotated by up to a few degrees to look at the effects of skew angles on envelope and emittance.

Many simulations have been carried out in support of both the phase I and phase II HCX [12], [13]. Examples include WARP code simulations of the non-linear multipole fields intrinsic to the prototype superconducting magnets, imperfectly aligned quadrupoles with small but finite rotation angles and displacements, finite initial

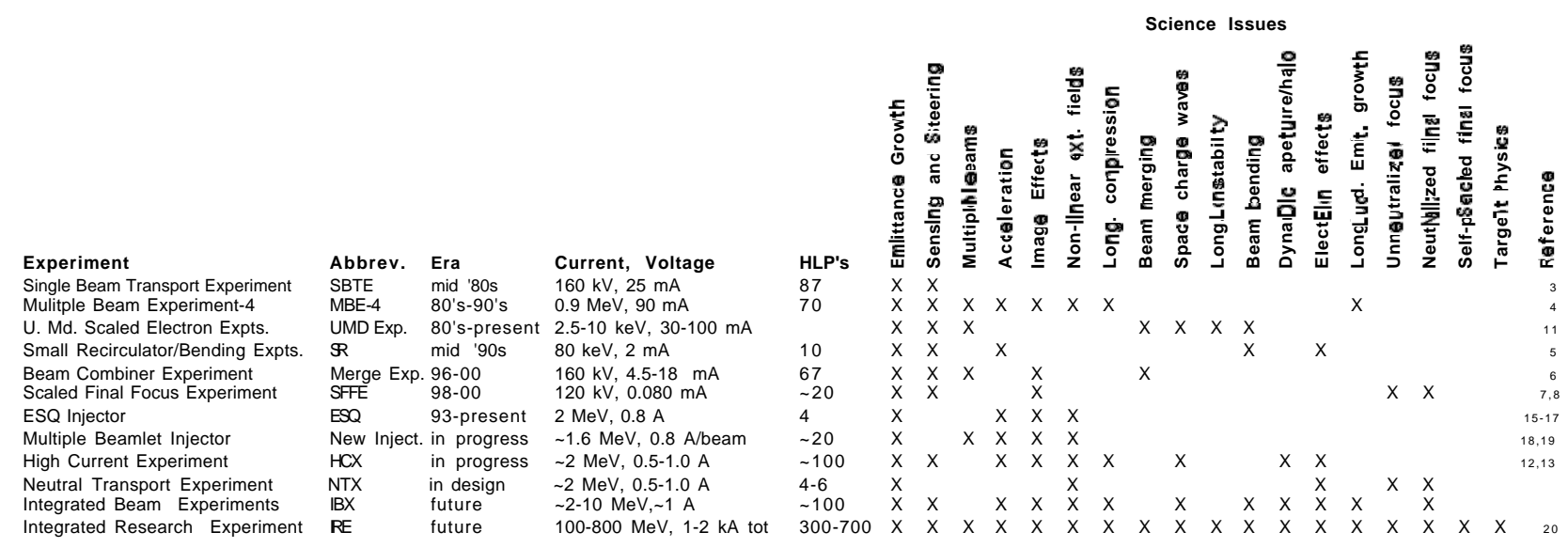

Table 1: Summary of Experiments in HIF Program 
displacements of the beam, and finite initial mismatches.

Several magnets are being designed for use in the HCX, (with the technology developed highly relevant to future accelerators including the Integrated Research Experiment). An array of 21 pulsed normal magnets was designed. An array which included 4 of these magnets has been pulsed approximately 80,000 times. Pulsed magnets would not be used in a driver (because the overall efficiency of the accelerator would not be acceptable for a power plant application) but, because of their cost advantage over superconducting magnets, pulsed magnets may be of value in an IRE or nearer-term experiment.

Two superconducting prototypes have been developed [14]. Both have circular apertures and square outer cross section, which would make them easily adaptable into a multi-beam array. One magnet uses rectangular Rutherford cables in a racetrack configuration of two layers, whereas the second group uses six layers of circular cables placed into grooves in a plastic matrix. Both prototypes have reached at least $90 \%$ of their theoretical maximum gradient, which is well above the requirements for the HCX. A selection of the baseline option will be made within a year.

The existing ESQ (which will be the front end to the HCX) has been found to have a current density distribution at its exit which is peaked near the edges[15]. The cause appears to be spherical aberration. Reduction of the radius of the source from $8.5 \mathrm{~cm}$ to $5 \mathrm{~cm}$ and modifications to the extraction electrode have, in simulations, reduced the nonuniformity and produced a more elliptical beam shape.

The other major VNL HIF experimental research project is design of a cost-effective injector. As pointed out earlier, multiple beams are required to transport the total charge required by the target. One way of doing this is to make a large diameter source that matches the maximum current limit of the ESQ transport channel. The ESQ Injector [17] is an example of this approach.

Another method, now being considered [18], is to merge hundreds of mm-scale beamlets into a single macro-beam. (There would still be tens to hundreds of macro-beams in the accelerator.) This merging-beamlet approach has the potential to reduce both the transverse and longitudinal dimensions of a multi-beam injector. The ChildLangmuir law for a diode relates the current density $\mathrm{J}$ and voltage $V$ across a gap of length $d$ according to $J \sim V^{3 / 2} / d^{2}$. But breakdown voltages are proportional to $d$ (for short distances $<\sim 1 \mathrm{~cm}$ ) and roughly as $\mathrm{d}^{1 / 2}$ for larger distances $>\sim 1 \mathrm{~cm}$ ). So $\mathrm{J} \sim \mathrm{V}^{-5 / 2}$ (small d) or $\mathrm{J} \sim \mathrm{V}^{-1 / 2}$ (large d). In either case the current density increases as the voltage decreases, whereas the total current goes as $\mathrm{V}^{3 / 2}$ (since $\mathrm{d}$ scales with the radius of the source). The beam brightness $\sim$ current/emittance $^{2} \sim \mathrm{J} / \mathrm{T}$ where $\mathrm{T}$ is the source temperature; thus high current density translates to high brightness. Low $\mathrm{V}$ implies high current density but to get the required high current many beamlets $(\sim 100$ 's per beam) are required. This approach is being both simulated using WARP and explored experimentally using a new $500 \mathrm{kV}$ source test stand now being completed at LLNL [18], [19].

Because of the large radius of the low current density source, the beamlines in a multiple beam injector must converge as they proceed from the source to the transport region. To bend the beam gradually, and decrease the beam radius from source to transport region, a large distance is required to match the beam to the ESQ transport channel. With the multiple beamlet approach all of the beams can feed directly into the transport channel, reducing substantially both length and radius. Furthermore, the beams can start out elliptical, minimizing the manipulations, in the matching section. This would be especially attractive for an IRE, where the front end is a much larger fraction of the cost than it is for a driver.

The final VNL experiment, now in the design stage, is a "Neutralized Transport Experiment" or NTX, the purpose of which is to examine neutralized final focus of higher perveance beams. (This has been designed to operate at the end of the MBE-4 or at the end of the High Current Experiment). The experiment consists of a number of magnetic quadrupoles that form the final focus, followed by a short drift, corresponding to propagation in the chamber. An rf source (now being readied at PPPL) ionizes the plasma, and so the effect of plasma at various locations within the chamber can be explored. This will be a flexible experiment allowing the experimental variation of plasma densities and gradients. Also, the large perveance planned for this experiment implies large final focusing angles, and therefore larger geometric aberrations. The question of whether octupoles can correct for the third order aberrations will be addressed experimentally on the NTX.

\subsection{Next Stage: Integrated Experiments}

After the HCX or as part of the later stages of HCX, the program envisions a near-term Integrated Beam Experiment (IBX) in which nearly all of the components of a driver are put into place. This would include injection, acceleration, longitudinal compression, and final focus, with a driver scale beam. The focus is on integration and validation. In August 2001, a workshop will be held with the goal of systematically determining the science goals of IBX and beginning to scope out the accelerator parameters. The IBX will enable comparisons of experimental data with source-to-target simulations, using WARP in the accelerator and LSP in the chamber.

The IBX will lay the ground work for the single final step between itself and a demonstration Inertial Fusion Energy test facility. That intermediate step is known as the Integrated Research Experiment (IRE). 
The goals of the IRE go beyond just a thorough understanding of high-intensity beam physics [20]. The basic overriding principle is that, together with the knowledge gained in the DOE Defense Programs target physics program including the National Ignition Facility (NIF) and supporting technology programs, the IRE will give a basis to proceed to an IFE Engineering Test Facility. Several areas of target physics (particularly those unique to ions [as opposed to lasers]) will begin being explored. (It is possible that hydrodynamic motion during the initial "foot" phase of the pulse could be investigated.) Examination of a variety of chamber transport modes, including self-pinched modes that are not accessible to the IBX, will be another major goal of the IRE. Further, chamber transport issues, particularly the interaction of beams with the liquid walls (Flibe) that are now favored to shield the solid walls of the target chamber, will be a research goal of the IRE. These goals dictate the scale of the facility to be tenth scale of a driver in ion energy, and in the 30-300 $\mathrm{kJ}$ range in pulse energy.

In the meantime, our information about the IRE and drivers comes from simulations and theory. Recently there has been substantial progress made in simulations and theory of the accelerator, driver and drift compression section [21-25], as well as detailed simulations and theory of chamber transport [26, 27], which include a number of plasma neutralization scenarios.

\section{SUMMARY AND CONCLUSIONS}

The Heavy Ion Fusion IFE program is transitioning from scaled experiments to experiments with beams that are driver scale in line charge density and pulse duration. The main scientific issues for the driver are maintenance of high brightness beams, and production and focusing of a highly neutralized beam in the chamber. WARP3D simulations of the accelerator and LSP simulations of the chamber, together with perturbative of (BEST) simulations, theory and lower-dimensional simulations, explore and validate the physics of near-term experiments, a mid-term IRE and longer-term driver beams. The current experimental emphasis of the HIF program is on HCX, advanced injector concepts, and NTX Final Focus experiments, with the IBX and IRE to follow.

\section{REFERENCES}

[1] D. A. Callahan-Miller and M. Tabak, "Progress in Target Physics and Design for Heavy Ion Fusion," Phys. of Plasmas, 7, 2083 (2000).

[2] W. Meier, A. Faltens, R. O. Bangerter, "An integrated systems model for heavy ion drivers," Nucl. Inst. and Meth. In Phys. Research, A., 415, 249, (1998).

[3] M.G Tiefenback,. and D. Keefe, "Measurements of Stability Limits for a Space Charge Dominated Ion Beam in a Long A.G. Transport Channel," IEEE Transactions on Nuclear Science, NS-32, 2483 (1985).

[4] W.M. Fawley, T. Garvey, S. Eylon, E.Henestroza, A. Faltens, T.J. Fessenden, K. Hahn, and L. Smith, "Beam Dynamics Studies with the Heavy Ion Linear Induction Accelerator MBE-4," Phys. Plasmas 4, 880 (1997).

[5] L. Ahle et al, "Results from the recirculator project at

LLNL,'Nuclear Instruments and Methods in Physics Research A, 464, 557 (2001).
[6] P.A. Seidl et al, "Progress on the scaled beam combining experiments at LBNL," Nucl. Instr. and Methods in Physics Research A, 415, 243 (1998).

[7] S. MacLaren, E. Henestroza, A. Faltens, G. Ritchie, and P.Seidl, "Results from the Scaled Final Focus Experiment," Nucl. Inst. and Meth. in Phys. Res. A 464, 126, (2001).

[8] D. Welch and D. Rose, "Beam,Neutralization in the Scaled Final

Focus Experiment," Proceedings of the 2001 Particle Accelerator Conference (RPAH068), (2001).

[9]A. Tauschwitz et al, "Plasma lens focusing and plasma channel transport for heavy ion fusion," Fusion Engineering and Design, 32-33, 493, (1996)

[10] P.A. Seidl et al,"Experiments at the Virtual National Laboratory for Heavy Ion Fusion," )", Nucl. Inst. and Meth. In Phys. Research, A., 415, 369, (2001).

[11] M. Reiser, et al, "The Maryland Electron Ring for Investigation of Space Charge Dominated Beams in a Circular FODO lattice," Proceedings of the 1999 Particle Acclerator Conference, NY p. 234 (1999). Also P. Oshea et al, "The Univ. of Md. Electron Ring (UMER)", Nucl. Inst. and Meth. In Phys. Research, A., 415, 646, (2001).

[12] P. A. Seidl et al, "Overview of the Scientific Objectives of the High Current Experiment for Heavy-Ion Fusion," Proceedings of the 2001 Particle Accelerator Conference (RPAH037), (2001).

[13] ] C.M. Celata, D.P. Grote, I. Haber, "PIC Simulations of Beam Dynamics Experiments on High Current Experiment," Proceedings of the 2001 Particle Accelerator Conference (RPAH033), (2001).

[14] G-L Sabbi et al, "Development of Superconducting Quadrupoles

for Heavy Ion Fusion," Proceedings of the 2001 Particle Accelerator

Conference (RPPH088), (2001).

[15] F. Bieniosek, E. Henestroza, J.W. Kwan,”2 MV Injector for

HCX," Proceedings of the 2001 Particle Accelerator Conference (WPAH010), (2001).

[16] E. Henestroza et al, "Beam Dynamics Studies of the Injector and Matching Section for HCX," Proceedings of the 2001 Particle

Accelerator Conference (RPAH035), (2001).

[17] S.S. Yu, S. Eylon, E. Henestroza, C. Peters, L. Reginato, A. Tauschwitz, D. Grote, and F. Deadrick, " 2 MV injector as the Elise front-end and as an experimental facility," Fusion Engineering and Design 32-33, 317 (1996)

[18] J.W. Kwan et al,"Design of a Compact High Current Injector for Heavy Ion Fusion," Proceedings of the 2001 Particle Accelerator Conference (WPAH013), (2001).

[19] L. Ahle et al,"Initial Experiments of RF Gas Plasma Source for Heavy Ion Fusion," Proceedings of the 2001 Particle Accelerator Conference (FOAC005), (2001).

[20] J.J. Barnard et al,"’lanning for a Heavy Ion Fusion Integrated Research Experiment," ," Nucl. Instr. and Methods in Physics

Research A 464, 621 (2001).

[21]I. Haber et al, "Recent Progress in Heavy Ion Fusion Simulations," "Nuclear Instruments and Methods in Physics Research A, 464, 343, (2001).

[22] A. Friedman et al, "Beam Simulations for IRE and Driver," "Nuclear Instruments and Methods in Physics Research A, 464, 653 (2001).

[23]M. de Hoon, E.P. Lee, J.J. Barnard,'Drift Compression of Space Charge Dominated Beams," Proceedings of the 2001 Particle Accelerator Conference (FOAC006), (2001).

[24] E.P. Lee and J.J. Barnard,"Design Strategies for Final Pulse Compression with Bending for Heavy Ion Fusion Drivers," Proceedings of the 2001 Particle Accelerator Conference (RPAH036), (2001).

[25] C. Jun et al, "Study of Drift Compression for Heavy Ion Fusion,"

Proceedings of the 2001 Particle Accelerator Conference (TPPH043), (2001).

[26] D.Welch et al,"Simulation of Self-Pinched Chamber Transport of Ions for Heavy Ion Fusion," Proceedings of the 2001 Particle

Accelerator Conference (RPAH067), (2001).

[27] I. Kaganovich, "Nonlinear Charge and Current Neutralization for an Intense Ion Beam Pulse Propagating Through a Background

Plasma," Proceedings of the 2001 Particle Accelerator Conference (TPPH317), (2001).

[28] H. Wollnik et al., "HIBALL-II: An Improved Conceptual Heavy Ion Driven Fusion Reactor Study," KfK-3840, FPA-84-4, UWFDM625, Karlsruhe, July, 1985. 